\title{
Wilson Bases and Ultradistributions
}

\author{
Nenad Teofanov
}

check for

updates

Citation: Teofanov, N. Wilson Bases and Ultradistributions. Axioms 2021, 10, 241. https://doi.org/10.3390/ axioms10040241

Academic Editor:

Palle E. T. Jorgensen

Received: 3 September 2021

Accepted: 22 September 2021

Published: 28 September 2021

Publisher's Note: MDPI stays neutral with regard to jurisdictional claims in published maps and institutional affiliations.

Copyright: (c) 2021 by the authors. Licensee MDPI, Basel, Switzerland. This article is an open access article distributed under the terms and conditions of the Creative Commons Attribution (CC BY) license (https:// creativecommons.org/licenses/by/ $4.0 /)$.
Department of Mathematics and Informatics, University of Novi Sad, 21000 Novi Sad, Serbia; nenad.teofanov@dmi.uns.ac.rs

Abstract: We provide a characterization of the Gelfand-Shilov-type spaces of test functions and their dual spaces of tempered ultradistributions by means of Wilson bases of exponential decay. We offer two different proofs and extend known results to the Roumieu case.

Keywords: Wilson bases; coorbit spaces; short-time Fourier transform; modulation spaces; GelfandShilov spaces

MSC: 42C15; 46F05; 41A58

\section{Introduction}

Wilson bases were constructed by I. Daubechies, S. Jaffard, and J. Journé in [1] to overcome constraints arising from the Balian-Low theorem, and soonafter shown to be unconditional bases for modulation spaces, see [2]. By combining the Wilson bases and tools from a time-frequency analysis, approximate diagonalization of different classes of pseudodifferential operators is obtained in [3,4]. Wilson bases of Meyer type were used in the study of gravitational waves, cf. [5,6]. We refer to [7] for recent construction of orthonormal Wilson bases in the multidimensional case, which overcomes a deficiency of the tensor product construction used in, e.g., [4,8].

Gelfand-Shilov spaces were initially introduced for the analysis of solutions of certain parabolic initial-value problems [9] and thereafter applied in different contexts when precise estimates of global decay and regularity are needed, see [10] for an overview. Recently, Hermite expansions of Fourier transform invariant Gelfand-Shilov spaces, and more generally Pilipović spaces, are considered in [11], see also [12,13].

In this paper, we give a description of Gelfand-Shilov spaces and their dual spaces of tempered ultradistributions in terms of Wilson bases. This extends some results from [14] given for Beurling type Gelfand-Shilov spaces. Both Wilson bases and Hermite functions are orthonormal bases for $L^{2}\left(\mathbb{R}^{d}\right)$ consisting of functions that are well localized in phasespace (time-frequency plane). From such perspective, our results are expected. However, due to the specific structure of Wilson bases, the proofs are based on entirely different arguments than those related to the Hermite basis, which utilize recursive relation between Hermite functions and the fact that they are eigenfunctions of the harmonic oscillator.

Instead, we apply the powerful general theory of coorbit spaces $[15,16]$. The key auxiliary result is the fact that Wilson bases are unconditional bases for coorbit spaces [2]. We modify and simplify the approach from [14] related to the Beurling case and provide detailed proofs since the more involved Roumieu case contains nontrivial modifications of arguments given there. As a consequence of our results, we recover the well-known relation between Gelfand-Shilov spaces and modulation spaces. Furthermore, if that relation is taken for granted, we give an alternative proof of our main results without an explicit reference to coorbit spaces.

Since both proofs are essentially based on the exponential decay of elements of Wilson bases and asymptotic behavior of the STFT, the techniques from the present paper can be modified to include other time-frequency representations and also more general (for exam- 
ple anisotropic) spaces of test functions and their distribution spaces. Such investigations are out of the scope of the present paper and will be the subject of our future work.

We end the introduction by recalling basic notation that will be used in the sequel.

Notation

Operators of translations and modulations of a given function $f$ are, respectively, given by $T_{x} f(\cdot)=f(\cdot-x)$ and $M_{y} f(\cdot)=e^{2 \pi i y \cdot} f(\cdot), x, y \in \mathbb{R}^{d}$. The notation $A \hookrightarrow B$ means that the topological spaces $A$ and $B$ satisfy $A \subseteq B$ with continuous embeddings. We write $A(\theta) \lesssim B(\theta), \theta \in \Omega$, if there is a constant $c>0$ such that $A(\theta) \leq c B(\theta)$ for all $\theta \in \Omega$.

The scalar product in $L^{2}\left(\mathbb{R}^{d}\right)$ is given by

$$
\langle f, g\rangle=\int_{\mathbb{R}^{d}} f(x) \overline{g(x)} d x,
$$

and $\|\cdot\|^{2}=\langle\cdot, \cdot\rangle$.

The Fourier transform of an absolutely integrable function $f$ is given by

$$
\hat{f}(\xi)=\mathcal{F} f(\xi)=\int_{\mathbb{R}^{d}} e^{-2 \pi i \xi x} f(x) d x, \xi \in \mathbb{R}^{d} .
$$

It extends uniquely to a unitary operator on $L^{2}\left(\mathbb{R}^{d}\right)$.

Let $\phi \in L^{2}\left(\mathbb{R}^{d}\right)$ be fixed. Then the short-time Fourier transform (STFT) $V_{\phi} f$ of $f \in$ $L^{2}\left(\mathbb{R}^{d}\right)$ with respect to the window function $\phi$ is defined by

$$
\begin{aligned}
& V_{\phi} f(x, \xi)=\mathcal{F}(f \overline{\phi(\cdot-x)})(\xi)=\left(f \cdot T_{x} \bar{\phi}\right)^{\wedge}(\xi) \\
&=\int_{\mathbb{R}^{d}} f(y) \overline{\phi(y-x)} e^{-2 \pi i \xi y} d y=\left\langle f, M_{\xi} T_{x} \phi\right\rangle, \quad x, \xi \in \mathbb{R}^{d} .
\end{aligned}
$$

Let $f_{1}, f_{2}, \phi_{1}, \phi_{2} \in L^{2}\left(\mathbb{R}^{d}\right)$. Then $V_{\phi_{j}} f_{j} \in L^{2}\left(\mathbb{R}^{2 d}\right), j=1,2$, and it satisfies the orthogonality relation ([Theorem 3.2.1] in [17]):

$$
\left\langle V_{\phi_{1}} f_{1}, V_{\phi_{2}} f_{2}\right\rangle=\left\langle\phi_{2}, \phi_{1}\right\rangle\left\langle f_{1}, f_{2}\right\rangle,
$$

whence $\left\|V_{\phi} f\right\|=\|\phi\| \cdot\|f\|$. The following fundamental identity of the time-frequency analysis $([17,18])$ is often used:

$$
V_{\phi} f(x, \xi)=e^{-2 \pi i x \cdot \xi} V_{\widehat{\phi}} \widehat{f}(\xi,-x), \quad x, \xi \in \mathbb{R}^{d} .
$$

By $\Sigma_{1}\left(\mathbb{R}^{d}\right)$ we denote the Gelfand-Shilov space of smooth functions given by:

$$
f \in \Sigma_{1}\left(\mathbb{R}^{d}\right) \Leftrightarrow\left\|f(x) e^{h \cdot|x|}\right\|_{L^{\infty}}<\infty \text { and }\left\|\hat{f}(\omega) e^{h \cdot|\omega|}\right\|_{L^{\infty}}<\infty, \quad \forall h>0,
$$

and its dual space is denoted by $\Sigma_{1}^{\prime}\left(\mathbb{R}^{d}\right)$.

If $\phi \in \Sigma_{1}\left(\mathbb{R}^{d}\right)$, then $\overline{M_{\xi} T_{x} \phi} \in \Sigma_{1}\left(\mathbb{R}^{d}\right)$, so by (1) it follows that the STFT can be extended to $\Sigma_{1}^{\prime}\left(\mathbb{R}^{d}\right)$, and restricted to $\Sigma_{1}\left(\mathbb{R}^{d}\right)$.

\section{Preliminaries}

In this section, we recall the Wilson bases, weight functions, coorbit spaces and Gelfand-Shilov type spaces. We also prove some auxiliary results (Lemmas 1 and 2 and Theorem 2), which will be used in Sections 3 and 4.

\subsection{Wilson Bases}

Following the idea of K. Wilson [19], Daubechies, Jaffard and Journe constructed a real-valued function $\psi$ such that

$$
|\psi(x)| \leq C e^{-a|x|}, \quad|\hat{\psi}(\xi)| \leq C e^{-b|\xi|} \quad x, \xi \in \mathbb{R},
$$


for some constants $a, b, C>0$, and obtain an orthonormal basis (ONB) $\left\{\psi_{l, n}\right\}_{l \in \mathbb{N}_{0}, n \in \mathbb{Z}}$, of $L^{2}(\mathbb{R})$, where

$$
\begin{aligned}
& \psi_{0, n}(x)=\quad T_{n} \psi(x), \\
& \psi_{l, n}(x)=\sqrt{2} \Re\left(M_{l} T_{n / 2} \psi(x)\right), \quad l+n \in 2 \mathbb{Z}, l \neq 0, \\
& \psi_{l, n}(x)=\sqrt{2} \Im\left(M_{l} T_{n / 2} \psi(x)\right), \quad l+n \in 2 \mathbb{Z}+1, l \neq 0,
\end{aligned}
$$

see [1]. From (5), it follows that

$$
\left|\psi_{l, n}(x)\right| \leq C e^{-a|x|}, \quad\left|\hat{\psi}_{l, n}(\xi)\right| \leq C e^{-b|\xi|}, \quad x, \xi \in \mathbb{R},(l, n) \in \mathbb{N}_{0} \times \mathbb{Z},
$$

for some constants $a, b, C>0$ depending on $l$ and $n$, and $\left\{\psi_{l, n}\right\}$ is therefore called the Wilson basis of exponential decay.

Equivalently, (6) can be written as $\psi_{0, n}=T_{n} \psi$ and

$$
\psi_{l, n}(x)= \begin{cases}\sqrt{2} \cos 2 \pi l x \psi\left(x-\frac{n}{2}\right), & l+n \in 2 \mathbb{Z}, l \neq 0, \\ \sqrt{2} \sin 2 \pi l x \psi\left(x-\frac{n}{2}\right), & l+n \in 2 \mathbb{Z}+1, l \neq 0 .\end{cases}
$$

Moreover, following Gröchenig [17], we may rewrite (6) as

$$
\begin{aligned}
& \psi_{0, n}(x)=T_{n} \psi(x), \quad \text { and } \\
& \qquad \psi_{l, n}(x)=\frac{1}{\sqrt{2}} T_{n / 2}\left(M_{l}+(-1)^{n+l} M_{-l}\right) \psi(x), \quad(l, n) \in \mathbb{N} \times \mathbb{Z} .
\end{aligned}
$$

To obtain an orthonormal basis of $L^{2}\left(\mathbb{R}^{d}\right)$, Tachizawa in [4] considered the $d$-dimensional Wilson basis given by the tensor product

$$
\psi_{l, n}(x)=\psi_{l_{1}, n_{1}}\left(x_{1}\right) \otimes \psi_{l_{2, n_{2}}}\left(x_{2}\right) \otimes \cdots \otimes \psi_{l_{d}, n_{d}}\left(x_{d}\right),
$$

$x=\left(x_{1}, x_{2}, \ldots, x_{d}\right) \in \mathbb{R}^{d}, l=\left(l_{1}, l_{2}, \ldots, l_{d}\right) \in \mathbb{N}_{0}^{d}, n=\left(n_{1}, n_{2}, \ldots, n_{d}\right) \in \mathbb{Z}^{d}$. If $\left\{\psi_{l_{k}, n_{k}}\left(x_{k}\right)\right\}$, $k=1, \ldots, d$, are Wilson bases of exponential decay, then we have

$$
\left|\psi_{l, n}(x)\right| \leq C e^{-a|x|}, \quad\left|\hat{\psi}_{l, n}(\xi)\right| \leq C e^{-b|\xi|} \quad x, \xi \in \mathbb{R}^{d},(l, n) \in \mathbb{N}_{0}^{d} \times \mathbb{Z}^{d},
$$

for some constants $a, b, C>0$, depending on $l$ and $n$.

The tensor product Wilson bases are $2^{d}$-modular, i.e., their elements have $2^{d}$ peaks in frequency, which may have undesirable consequences in applications, see [7] for details. That motivated Bownik et al. [7] to construct a family of orthonormal Wilson bases with $2^{k}$-modular covering of the frequency domain with $k=1, \ldots, d$. The tensor product Wilson bases turned out to be the special case of their construction.

\subsection{Weight Functions}

A weight on $\mathbb{R}^{d}$ is a positive function $\omega \in L_{\text {loc }}^{\infty}\left(\mathbb{R}^{d}\right)$ such that $1 / \omega \in L_{l o c}^{\infty}\left(\mathbb{R}^{d}\right)$. The weight $\omega$ on $\mathbb{R}^{d}$ is called moderate if there is a positive locally bounded function $v$ on $\mathbb{R}^{d}$ such that

$$
\omega(x+y) \leq C \omega(x) v(y), \quad x, y \in \mathbb{R}^{d},
$$

for some constant $C \geq 1$. If $\omega$ and $v$ are weights on $\mathbb{R}^{d}$ such that (9) holds, then $\omega$ is also called v-moderate. If $v$ can be chosen as a polynomial, then $\omega$ is called a weight of polynomial type. The set of all moderate weights on $\mathbb{R}^{d}$ is denoted by $\mathcal{P}_{E}\left(\mathbb{R}^{d}\right)$.

The weight $v$ on $\mathbb{R}^{d}$ is called submultiplicative, if it is even and (9) holds for $\omega=v$. From now on, $v$ always denotes a submultiplicative weight if nothing else is stated. In 
particular, if (9) holds and $v$ is submultiplicative, then it follows by straight-forward computations that

$$
\begin{gathered}
\frac{\omega(x)}{v(y)} \lesssim \omega(x+y) \lesssim \omega(x) v(y), \\
v(x+y) \lesssim v(x) v(y) \text { and } v(x)=v(-x), \quad x, y \in \mathbb{R}^{d} .
\end{gathered}
$$

If $\omega$ is a moderate weight on $\mathbb{R}^{d}$, then there is a submultiplicative weight $v$ on $\mathbb{R}^{d}$ such that (9) and (10) hold, see $[20,21]$. Moreover if $v$ is submultiplicative on $\mathbb{R}^{d}$, then

$$
1 \lesssim v(x) \lesssim e^{r|x|}
$$

for some constant $r>0$ (cf. [20]). In particular, if $\omega$ is moderate, then

$$
\omega(x+y) \lesssim \omega(x) e^{r|y|} \text { and } e^{-r|x|} \lesssim \omega(x) \lesssim e^{r|x|}, \quad x, y \in \mathbb{R}^{d}
$$

for some $r>0$.

We will consider only weight functions $w$ satisfying Beurling-Domar's non-quasianalyticity condition

$$
\sum_{n=1}^{\infty} n^{-2} \log \omega(n x, n y)<\infty, \quad x, y \in \mathbb{R}^{d} .
$$

The most important examples of weight functions that satisfy (13) are $(1+|x|)^{s}$, $(1+|y|)^{s},(1+|x|+|y|)^{s}, e^{s\left(|x|^{\gamma}+|y|^{\gamma}\right)}, x, y \in \mathbb{R}^{d}, s \geq 0, \gamma \in(0,1)$.

If $\omega \in \mathcal{P}_{E}\left(\mathbb{R}^{d}\right)$ then the weighted $L^{2}\left(\mathbb{R}^{d}\right)$ space, $L_{\omega}^{2}\left(\mathbb{R}^{d}\right)$ is given by

$$
f \in L_{\omega}^{2}\left(\mathbb{R}^{d}\right) \Leftrightarrow\|f\|_{L_{\omega}^{2}}=\|f \omega\|_{L^{2}}<\infty
$$

\subsection{Coorbit Spaces}

For the purpose of this paper, we focus our attention to subexponential weights of the form $\omega_{h, s}(\cdot)=e^{h|\cdot|^{1 / s}}, s>1, h \geq 0$. Such weights satisfy the condition (13).

Definition 1. Let there be given $s>1, h \geq 0$ and $\phi \in \Sigma_{1}\left(\mathbb{R}^{d}\right) \backslash 0$. The coorbit space $\mathcal{C} o Y^{h, s}\left(\mathbb{R}^{d}\right)$ is defined by

$$
\begin{aligned}
& \mathcal{C o Y}^{h, s}\left(\mathbb{R}^{d}\right)=\{f \in \Sigma_{1}^{\prime}\left(\mathbb{R}^{d}\right) \mid \\
&\left.\|f\|_{\mathcal{C o Y} Y, s} \equiv \int_{\mathbb{R}^{d}}\left(\int_{\mathbb{R}^{d}}\left|V_{\phi} f(x, \xi)\right|^{2} d x\right) e^{2 h|\xi|^{1 / s}} d \xi<\infty\right\} .
\end{aligned}
$$

In other words, $f \in \mathcal{C o}_{o} Y^{h, s}\left(\mathbb{R}^{d}\right)$ if $F(\xi)=\left\|V_{\phi} f(\cdot, \xi)\right\|_{L^{2}} \in L_{\omega_{h, s}}^{2}\left(\mathbb{R}^{d}\right)$ (cf. (14)).

This terminology (and notation) is justified by the general theory of coorbit spaces developed in [15,16], see also [22] for a more recent survey.

From the results given there, it follows that $\mathcal{C o}_{0} Y^{h, s}\left(\mathbb{R}^{d}\right)$ is a Banach space invariant under translations, modulations, and complex conjugations. Moreover, $\mathcal{C} o Y^{h, s}\left(\mathbb{R}^{d}\right)$ is independent on the choice of $\phi \in \Sigma_{1}\left(\mathbb{R}^{d}\right) \backslash 0$, see e.g., [Proposition 3.2 (ii)] in [22].

We will use the following simple results.

Lemma 1. Let $s>1, h \geq 0$, and $\phi \in \Sigma_{1}\left(\mathbb{R}^{d}\right) \backslash 0$. Then $\hat{f} \in \mathcal{C} o Y^{h, s}\left(\mathbb{R}^{d}\right)$ if and only if

$$
\int_{\mathbb{R}^{d}}\left(\int_{\mathbb{R}^{d}}\left|V_{\phi} f(y, \eta)\right|^{2} d \eta\right) e^{2 h|y|^{1 / s}} d y<\infty .
$$


Proof. Since different elements $\phi \in \Sigma_{1}\left(\mathbb{R}^{d}\right) \backslash 0$ give rise to the same space $\mathcal{C} o Y^{h, s}\left(\mathbb{R}^{d}\right)$ we may take the Gaussian $\phi(x)=\hat{\phi}(x)=e^{-\pi x^{2}}$. By (3) and the change of variables we have:

$$
\begin{aligned}
& \int_{\mathbb{R}^{d}}\left(\int_{\mathbb{R}^{d}}\left|V_{\phi} \hat{f}(x, \xi)\right|^{2} d x\right) e^{2 h|\xi|^{1 / s}} d \xi \\
= & \int_{\mathbb{R}^{d}}\left(\int_{\mathbb{R}^{d}}\left|V_{\hat{\phi}} \hat{f}(x, \xi)\right|^{2} d x\right) e^{2 h|\xi|^{1 / s}} d \xi \\
= & \int_{\mathbb{R}^{d}}\left(\int_{\mathbb{R}^{d}}\left|V_{\phi} f(-\xi, x)\right|^{2} d x\right) e^{2 h|\xi|^{1 / s}} d \xi \\
= & \int_{\mathbb{R}^{d}}\left(\int_{\mathbb{R}^{d}}\left|V_{\phi} f(y, \eta)\right|^{2} d \eta\right) e^{2 h|y|^{1 / s}} d y .
\end{aligned}
$$

Thus $\hat{f} \in \mathcal{C}$ o $Y^{h, s}\left(\mathbb{R}^{d}\right)$ if and only if (16) holds true.

We write

$$
f \in \mathcal{F C} o Y^{h, s}\left(\mathbb{R}^{d}\right) \quad \text { if } \quad \hat{f} \in \mathcal{C} o Y^{h, s}\left(\mathbb{R}^{d}\right) .
$$

(According to the general theory of coorbit spaces it follows that $\mathcal{F C} o Y^{h, s}\left(\mathbb{R}^{d}\right)$ is a coorbit space as well.)

Lemma 2. Let there be given $s>1$ and $h \geq 0$. Then we have

(a) $f \in \mathcal{C} o Y^{h, s}\left(\mathbb{R}^{d}\right)$ if and only if $f(x) e^{h|x|^{1 / s}} \in L^{2}\left(\mathbb{R}^{d}\right)$.

(b) $f \in \mathcal{F} \mathcal{C o} Y^{h, s}\left(\mathbb{R}^{d}\right)$ if and only if $\hat{f}(\xi) e^{h|\xi|^{1 / s}} \in L^{2}\left(\mathbb{R}^{d}\right)$.

Proof. We again choose $\phi(x)=e^{-\pi x^{2}}$ in the definition of $\mathcal{C} o Y^{h, s}\left(\mathbb{R}^{d}\right)$ and follow the idea of the proof of [17] (Proposition 11.3.1). By (1) and the Plancherel theorem, we formally have

$$
\int_{\mathbb{R}^{d}}\left|V_{\phi} f(x, \xi)\right|^{2} d \xi=\int_{\mathbb{R}^{d}}|f(t)|^{2}|\phi(t-x)|^{2} d t .
$$

Since $e^{2 h|x|^{1 / s}}$ is a moderate weight, it follows that

$$
e^{-2 h|u|^{1 / s}} e^{2 h|t|^{1 / s}} \lesssim e^{2 h|t-u|^{1 / s}} \lesssim e^{2 h|t|^{1 / s}} e^{2 h|u|^{1 / s}}, \quad t, u \in \mathbb{R}^{d},
$$

cf. (10). Therefore,

$$
\begin{array}{r}
\int_{\mathbb{R}^{d}}|f(t)|^{2} e^{2 h|t|^{1 / s}} d t \int_{\mathbb{R}^{d}}|\phi(u)|^{2} e^{-2 h|u|^{1 / s}} d u \\
\lesssim \int_{\mathbb{R}^{d}} \int_{\mathbb{R}^{d}}|f(t)|^{2}|\phi(u)|^{2} e^{2 h|t-u|^{1 / s}} d t d u \\
=\int_{\mathbb{R}^{d}} \int_{\mathbb{R}^{d}}|f(t)|^{2}|\phi(t-x)|^{2} e^{2 h|x|^{1 / s}} d t d x \\
\lesssim \int_{\mathbb{R}^{d}}|f(t)|^{2} e^{2 h|t|^{1 / s}} d t \int_{\mathbb{R}^{d}}|\phi(u)|^{2} e^{2 h|u|^{1 / s}} d u
\end{array}
$$

and (a) follows.

Part (b) follows from (a) and (17).

From the general theory of coorbit spaces it follows that Wilson bases of exponential decay are unconditional bases for $\mathcal{C o}_{0} Y^{h, s}\left(\mathbb{R}^{d}\right)$ and $\mathcal{F C o} Y^{h, s}\left(\mathbb{R}^{d}\right)$, [2,14]. The precise statement is the following.

Theorem 1. Let there be given $s>1, h \geq 0$, and the Wilson basis of exponential decay $\left\{\psi_{l, n}\right\}_{l \in \mathbb{N}_{0}^{d}, n \in \mathbb{Z}^{d}}$. Let $\mathcal{C} 0 Y^{h, s}\left(\mathbb{R}^{d}\right)$ and $\mathcal{F C} O Y^{h, s}\left(\mathbb{R}^{d}\right)$ be given by (15) and (17), respectively. Then we have: 
(a) The Wilson basis $\left\{\psi_{l, n}\right\}_{l \in \mathbb{N}_{0}^{d}, n \in \mathbb{Z}^{d}}$ of exponential decay is an unconditional basis for the coorbit spaces $\mathcal{C} o Y^{h, s}\left(\mathbb{R}^{d}\right)$ and $\mathcal{F C} O Y^{h, s}\left(\mathbb{R}^{d}\right)$.

(b) Every function $f \in \mathcal{C}_{0} Y^{h, s}\left(\mathbb{R}^{d}\right)$ has the unique expansion

$$
f=\sum_{l \in \mathbb{N}_{0}^{d}, n \in \mathbb{Z}^{d}} c_{l, n} \psi_{l, n} \text { where } c_{l, n}=\left\langle f, \psi_{l, n}\right\rangle, \quad l \in \mathbb{N}_{0}^{d}, n \in \mathbb{Z}^{d},
$$

and

$$
\sum_{l \in \mathbb{N}_{0}^{d}}\left(\sum_{n \in \mathbb{Z}^{d}}\left|c_{l, n}\right|^{2}\right) e^{2 h|l|^{1 / s}}<\infty .
$$

(c) Every function $f \in \mathcal{F C}$ o $Y^{h, s}\left(\mathbb{R}^{d}\right)$ has the unique expansion of the form (18) and

$$
\sum_{l \in \mathbb{N}_{0}^{d}}\left(\sum_{n \in \mathbb{Z}^{d}}\left|c_{l, n}\right|^{2}\right) e^{2 h\left|\frac{n}{2}\right|^{1 / s}}<\infty .
$$

Proof. The proof is omitted since it follows the arguments given in the proof of Theorem 4 in [2], where polynomial type weights are considered instead, see also [4]. The subexponential type weights considered here are treated in [14], see in particular [Theorem 4.4, Remark 4.5] in [14].

\subsection{Gelfand-Shilov Spaces}

Gelfand and Shilov introduced the spaces of type $S$, for the analysis of solutions of certain parabolic initial-value problems. A comprehensive study of those spaces, which are afterward called Gelfand-Shilov spaces, is given in [9]. We focus our attention on the case when regularity and decay are controlled by the so-called Gevrey sequences $M_{p}=p !^{s}$, when $s>0$ and refer to, e.g., [23] for an overview of a more general situation.

Let $0<s$ be fixed. Then the (Fourier invariant) Gelfand-Shilov space $\mathcal{S}_{s}\left(\mathbb{R}^{d}\right)\left(\Sigma_{s}\left(\mathbb{R}^{d}\right)\right)$ of Roumieu type (Beurling type) consists of all $f \in C^{\infty}\left(\mathbb{R}^{d}\right)$ such that

$$
\|f\|_{\mathcal{S}_{s, h}} \equiv \sup _{\alpha, \beta \in \mathbb{N}^{d}} \sup _{x \in \mathbb{R}^{d}} \frac{\left|x^{\alpha} \partial^{\beta} f(x)\right|}{h^{|\alpha+\beta|}(\alpha ! \beta !)^{s}}
$$

is finite for some $h>0$ (for every $h>0$ ). The semi-norms $\|\cdot\|_{\mathcal{S}_{s, h}}$ induce an inductive limit topology for the space $\mathcal{S}_{s}\left(\mathbb{R}^{d}\right)$, and a projective limit topology for $\Sigma_{s}\left(\mathbb{R}^{d}\right)$. Thus, the former space becomes an LS space, while the latter space is an FS space (Fréchet-Schwartz space) under these topologies.

The space $\mathcal{S}_{s}\left(\mathbb{R}^{d}\right) \neq\{0\}\left(\Sigma_{s}\left(\mathbb{R}^{d}\right) \neq\{0\}\right)$, if and only if $s \geq \frac{1}{2}\left(s>\frac{1}{2}\right)$.

The Gelfand-Shilov distribution spaces $\mathcal{S}_{s}^{\prime}\left(\mathbb{R}^{d}\right)$ and $\Sigma_{s}^{\prime}\left(\mathbb{R}^{d}\right)$ (also known as spaces of tempered ultradistributions) are the dual spaces of $\mathcal{S}_{s}\left(\mathbb{R}^{d}\right)$ and $\Sigma_{s}\left(\mathbb{R}^{d}\right)$, respectively.

We have

$$
\begin{aligned}
\mathcal{S}_{1 / 2}\left(\mathbb{R}^{d}\right) & \hookrightarrow \Sigma_{s}\left(\mathbb{R}^{d}\right) \hookrightarrow \mathcal{S}_{s}\left(\mathbb{R}^{d}\right) \hookrightarrow \Sigma_{t}\left(\mathbb{R}^{d}\right) \\
& \hookrightarrow \mathcal{S}\left(\mathbb{R}^{d}\right) \hookrightarrow \mathcal{S}^{\prime}\left(\mathbb{R}^{d}\right) \hookrightarrow \Sigma_{t}^{\prime}\left(\mathbb{R}^{d}\right) \\
& \hookrightarrow \mathcal{S}_{s}^{\prime}\left(\mathbb{R}^{d}\right) \hookrightarrow \Sigma_{s}^{\prime}\left(\mathbb{R}^{d}\right) \hookrightarrow \mathcal{S}_{1 / 2}^{\prime}\left(\mathbb{R}^{d}\right), \quad \frac{1}{2}<s<t .
\end{aligned}
$$

The Fourier transform $\mathcal{F}$ extends uniquely to a homeomorphism on $\mathcal{S}_{s}^{\prime}\left(\mathbb{R}^{d}\right)$ and on $\Sigma_{s}^{\prime}\left(\mathbb{R}^{d}\right)$. Furthermore, $\mathcal{F}$ restricts to a homeomorphism on $\mathcal{S}_{s}\left(\mathbb{R}^{d}\right)$ and on $\Sigma_{s}\left(\mathbb{R}^{d}\right)$. Similar facts hold true when the Fourier transform is replaced by a partial Fourier transform.

The Fourier transform invariance of $\mathcal{S}_{s}\left(\mathbb{R}^{d}\right)$ and $\Sigma_{s}\left(\mathbb{R}^{d}\right)$ follows from the following result, which also gives a characterization of Gelfand-Shilov spaces in terms of coorbit spaces. 
Theorem 2. Let there be given $s>1$. The following conditions are equivalent:

(a) $f \in \mathcal{S}_{s}\left(\mathbb{R}^{d}\right)\left(f \in \Sigma_{s}\left(\mathbb{R}^{d}\right)\right) ;$

(b) There exists $h>0$ (for every $h>0$ )

$$
\sup _{x \in \mathbb{R}^{d}}|f(x)| e^{h|x|^{1 / s}}<\infty \text { and } \sup _{\xi \in \mathbb{R}^{d}}|\hat{f}(\xi)| e^{h|\xi|^{1 / s}}<\infty ;
$$

(c) There exists $h>0$ (for every $h>0$ ) such that

$$
f \in \mathcal{C o}_{0} Y^{h, s}\left(\mathbb{R}^{d}\right) \cap \mathcal{F C O} Y^{h, s}\left(\mathbb{R}^{d}\right) .
$$

Proof. (a) $\Leftrightarrow$ (b) is well known and holds for all $s>0,[24,25]$.

(b) $\Leftrightarrow$ (c). We first consider the Beurling case. By the $d$-dimensional version of [14] (Theorem 2.2), it follows that the sup-norms in (23) can be replaced by $L_{\omega_{k, s}}^{2}$-norms, i.e., for every $k>0$, we have

$$
\int_{\mathbb{R}^{d}}|f(x)|^{2} e^{2 k|x|^{1 / s}} d x<\infty \text { and } \int_{\mathbb{R}^{d}}|\hat{f}(\xi)|^{2} e^{2 k|\xi|^{1 / s}} d \xi<\infty .
$$

This, together with Lemma 2 gives

$$
f \in \Sigma_{s}\left(\mathbb{R}^{d}\right) \Leftrightarrow f \in \mathcal{C} o Y^{h, s}\left(\mathbb{R}^{d}\right) \cap \mathcal{F C} o Y^{h, s}\left(\mathbb{R}^{d}\right)
$$

for every $h>0$.

For the Roumieu case, by slight modifications of the proof of [Theorem 2.2] in [14], it also follows that (23) holds for some $h>0$ if and only if (24) holds for some $k>0$. Again, Lemma 2 implies that this is equivalent with $f \in \mathcal{C}_{0} Y^{h, s}\left(\mathbb{R}^{d}\right) \cap \mathcal{F C} 0 Y^{h, s}\left(\mathbb{R}^{d}\right)$ for some $h>0$, and the proof is finished.

Note that, since the equivalence between a) and b) in Theorem 2 holds even if $s=1$, if $\psi$ satisfies (5), then the Wilson basis elements $\psi_{l, n}, l \in \mathbb{N}_{0}^{d}, n \in \mathbb{Z}^{d}$, given by (6) belongs to $\mathcal{S}_{1}\left(\mathbb{R}^{d}\right)$, cf. (7).

The restriction $s>1$ when proving b) $\Leftrightarrow \mathrm{c}$ ) in Theorem 2 comes from Definition 1 . In fact, in the general theory of coorbit spaces, as presented in [15,16], an important role is played by BUPUs (bounded uniform partitions of unity) consisting of compactly supported smooth functions. In such setting, coorbit spaces consist of non-quasianalytic functions.

From the definitions of $\mathcal{C} o Y^{h, s}\left(\mathbb{R}^{d}\right), \mathcal{F} \mathcal{C} o Y^{h, s}\left(\mathbb{R}^{d}\right)$ and Theorem 2 (c) it follows that the Gelfand-Shilov spaces are essentially characterized by the decay estimates of the shorttime Fourier transform. Note that the estimates given in Proposition 1 below employ the sup-norm $\left(L^{\infty}\right.$-norm) whereas in Theorem 2 (c) the $L^{2}$-norm related to $\mathcal{C} o Y^{h, s}\left(\mathbb{R}^{d}\right)$ and $\mathcal{F} \mathcal{C} o Y^{h, s}\left(\mathbb{R}^{d}\right)$ is considered instead. (In fact, any $L^{p}$-norm $(1 \leq p \leq \infty)$ can be used, see [13].)

Proposition 1. Let $s \geq \frac{1}{2}\left(s>\frac{1}{2}\right), \phi \in \mathcal{S}_{s}\left(\mathbb{R}^{d}\right) \backslash 0\left(\phi \in \Sigma_{s}\left(\mathbb{R}^{d}\right) \backslash 0\right)$ and let $f$ be a GelfandShilov distribution on $\mathbb{R}^{d}$. Then the following is true:

(a) $f \in \mathcal{S}_{s}\left(\mathbb{R}^{d}\right)\left(f \in \Sigma_{s}\left(\mathbb{R}^{d}\right)\right)$, if and only if

$$
\left|V_{\phi} f(x, \xi)\right| \lesssim e^{-r\left(|x|^{\frac{1}{s}}+|\xi|^{\frac{1}{s}}\right)}, \quad x, \xi \in \mathbb{R}^{d},
$$

for some $r>0$ (for every $r>0$ ).

(b) $f \in \mathcal{S}_{s}^{\prime}\left(\mathbb{R}^{d}\right)\left(f \in \Sigma_{s}^{\prime}\left(\mathbb{R}^{d}\right)\right)$, if and only if

$$
\left|V_{\phi} f(x, \xi)\right| \lesssim e^{r\left(|x|^{\frac{1}{s}}+|\xi|^{\frac{1}{\sigma}}\right)}, \quad x, \xi \in \mathbb{R}^{d},
$$

for every $r>0$ (for some $r>0)$. 
We omit the proof since the first part follows from [24] (Theorem 2.7) and the second part from [11] (Proposition 2.2). See also [26] for related results.

From these investigations and by [21] (Theorem 2.3) it follows that the definition of the map $(f, \phi) \mapsto V_{\phi} f$ from $L^{2}\left(\mathbb{R}^{d}\right) \times L^{2}\left(\mathbb{R}^{d}\right)$ to $L^{2}\left(\mathbb{R}^{2 d}\right)$ is uniquely extendable to a continuous map from $\mathcal{S}_{s}^{\prime}\left(\mathbb{R}^{d}\right) \times \mathcal{S}_{S}^{\prime}\left(\mathbb{R}^{d}\right)$ to $\mathcal{S}_{s}^{\prime}\left(\mathbb{R}^{2 d}\right)$, and restricts to a continuous map from $\mathcal{S}_{s}\left(\mathbb{R}^{d}\right) \times \mathcal{S}_{s}\left(\mathbb{R}^{d}\right)$ to $\mathcal{S}_{s}\left(\mathbb{R}^{2 d}\right)$. The same conclusion holds with $\Sigma_{s}$ in place of $\mathcal{S}_{s}$, at each place. Therefore, Definition 1 can be appropriately modified to include ultradistributions $f \in \mathcal{S}_{s}^{\prime}\left(\mathbb{R}^{d}\right)$ (or $f \in \Sigma_{s}^{\prime}\left(\mathbb{R}^{d}\right)$ ), and we will use such extension from now on.

\section{Main Results}

In this section, we discuss Wilson base expansions in the context of Gelfand-Shilov spaces and their dual spaces of tempered ultradistributions.

Theorem 3. Let $s>1$ and let there be given a Wilson basis of exponential decay $\left\{\psi_{l, n}\right\}_{l \in \mathbb{N}_{0}^{d}, n \in \mathbb{Z}^{d}}$.

(a) If $f \in \mathcal{S}_{s}\left(\mathbb{R}^{d}\right)\left(f \in \Sigma_{s}\left(\mathbb{R}^{d}\right)\right)$ then

$$
f=\sum_{l \in \mathbb{N}_{0}^{d}} \sum_{n \in \mathbb{Z}^{d}}\left\langle f, \psi_{l, n}\right\rangle \psi_{l, n}
$$

with the unconditional convergence in $\mathcal{S}_{s}\left(\mathbb{R}^{d}\right)\left(\right.$ in $\Sigma_{s}\left(\mathbb{R}^{d}\right)$ ) and

$$
\sum_{l \in \mathbb{N}_{0}^{d}, n \in \mathbb{Z}^{d}}\left|c_{l, n}\right|^{2} e^{2 k(|n / 2|+|l|)^{1 / s}}<\infty, \text { for some (for all) } k \geq 0,
$$

where $c_{l, n}=\left\langle f, \psi_{l, n}\right\rangle, l \in \mathbb{N}_{0}^{d}, n \in \mathbb{Z}^{d}$.

(b) Conversely, if $\left(c_{l, n}\right)_{l \in \mathbb{N}_{0}^{d}, n \in \mathbb{Z}^{d}}$ is a (double) sequence such that

$$
\sum_{l \in \mathbb{N}_{0}^{d}, n \in \mathbb{Z}^{d}}\left|c_{l, n}\right|^{2} e^{2 k(|n / 2|+|l|)^{1 / s}}<\infty,
$$

for some (for all) $k \geq 0$, then there exists a function $f \in \mathcal{S}_{s}\left(\mathbb{R}^{d}\right)\left(f \in \Sigma_{s}\left(\mathbb{R}^{d}\right)\right)$ such that (27) holds with $c_{l, n}=\left\langle f, \psi_{l, n}\right\rangle, l \in \mathbb{N}_{0}^{d}, n \in \mathbb{Z}^{d}$.

Proof. (a) We prove the Roumieu case, since the Beurling case is given in [14] (Theorem 5.1 (a)). Let $f \in \mathcal{S}_{s}\left(\mathbb{R}^{d}\right)$. By Theorem 2 (c), we have that

$$
f \in \mathcal{C o}_{0} Y^{h, s}\left(\mathbb{R}^{d}\right) \cap \mathcal{F C} o Y^{h, s}\left(\mathbb{R}^{d}\right)
$$

for some $h>0$. Then, from Theorem 1 it follows that (19) and (20) hold for that constant $h>0$. Therefore,

$$
\begin{gathered}
\sum_{l \in \mathbb{N}_{0}^{d}, n \in \mathbb{Z}^{d}}\left|c_{l, n}\right|^{2} e^{2 \frac{h}{2}(|n / 2|+\mid l l)^{1 / s}} \\
\leq \sum_{l \in \mathbb{N}_{0}^{d}, n \in \mathbb{Z}^{d}}\left|c_{l, n}\right| e^{2 \frac{h}{2}|n / 2|^{1 / s}} \cdot\left|c_{l, n}\right| e^{2 \frac{h}{2}|l|^{1 / s}} \\
\leq\left(\sum_{l \in \mathbb{N}_{0}^{d}, n \in \mathbb{Z}^{d}}\left|c_{l, n}\right|^{2} e^{2 h|n / 2|^{1 / s}}\right)^{1 / 2} \cdot\left(\sum_{l \in \mathbb{N}_{0}^{d}, n \in \mathbb{Z}^{d}}\left|c_{l, n}\right|^{2} e^{2 h|l|^{1 / s}}\right)^{1 / 2}<\infty,
\end{gathered}
$$

so that

$$
\sum_{l \in \mathbb{N}_{0}^{d}, n \in \mathbb{Z}^{d}}\left|c_{l, n}\right|^{2} e^{2 k(|n / 2|+|l|)^{1 / s}}<\infty
$$

for $k=h / 2$. The unconditional convergence follows from Theorem 1 (a). 
To prove (b), we note that (28) obviously implies

$$
\sum_{l \in \mathbb{N}_{0}^{d}, n \in \mathbb{Z}^{d}}\left|c_{l, n}\right|^{2} e^{2 k|n / 2|^{1 / s}}<\infty,
$$

and

$$
\sum_{l \in \mathbb{N}_{0}^{d}, n \in \mathbb{Z}^{d}}\left|c_{l, n}\right|^{2} e^{2 k|l|^{1 / s}}<\infty .
$$

This, together with Theorem 1 gives

$$
f=\sum_{l \in \mathbb{N}_{0}^{d}} \sum_{n \in \mathbb{Z}^{d}} c_{l, n} \psi_{l, n} \in \mathcal{C} o Y^{h, s}\left(\mathbb{R}^{d}\right) \cap \mathcal{F C} o Y^{h, s}\left(\mathbb{R}^{d}\right),
$$

and since the Wilson basis is an ONB we have that $c_{l, n}=\left\langle f, \psi_{l, n}\right\rangle, l \in \mathbb{N}_{0}^{d}, n \in \mathbb{Z}^{d}$. Now, by Theorem 2, we conclude that $f \in \mathcal{S}_{\mathcal{S}}\left(\mathbb{R}^{d}\right)$, and the proof is finished.

Theorem 4. Let $s>1$ and let there be given a Wilson basis of exponential decay $\left\{\psi_{l, n}\right\}_{l \in \mathbb{N}_{0}^{d}, n \in \mathbb{Z}^{d}}$.

(a) Every $f \in \mathcal{S}_{s}^{\prime}\left(\mathbb{R}^{d}\right)\left(f \in \Sigma_{s}^{\prime}\left(\mathbb{R}^{d}\right)\right)$ has a unique expansion

$$
f=\sum_{l \in \mathbb{N}_{0}^{d}, n \in \mathbb{Z}^{d}}\left\langle f, \psi_{l, n}\right\rangle \psi_{l, n}
$$

in $\mathcal{S}_{S}^{\prime}\left(\mathbb{R}^{d}\right)\left(\right.$ in $\left.\Sigma_{s}^{\prime}\left(\mathbb{R}^{d}\right)\right)$ and

$$
\sum_{l \in \mathbb{N}_{0}^{d}, n \in \mathbb{Z}^{d}}\left|c_{l, n}\right|^{2} e^{-2 h(|n / 2|+|l|)^{1 / s}}<\infty,
$$

for every (for some) $h \geq 0$, where $c_{l, n}=\left\langle f, \psi_{l, n}\right\rangle, l \in \mathbb{N}_{0}^{d}, n \in \mathbb{Z}^{d}$.

(b) Conversely, if (29) holds for some sequence $\left(c_{l, n}\right)_{l \in \mathbb{N}_{0}^{d}, n \in \mathbb{Z}^{d}}$ and for every (for some) $h \geq 0$, then there exists $f \in \mathcal{S}_{s}^{\prime}\left(\mathbb{R}^{d}\right)\left(f \in \Sigma_{s}^{\prime}\left(\mathbb{R}^{d}\right)\right)$ such that

$$
f=\sum_{l \in \mathbb{N}_{0}^{d}, n \in \mathbb{Z}^{d}} c_{l, n} \psi_{l, n}
$$

in $\mathcal{S}_{S}^{\prime}\left(\mathbb{R}^{d}\right)\left(\right.$ in $\left.\Sigma_{s}^{\prime}\left(\mathbb{R}^{d}\right)\right)$

For the proof of Theorem 4, we need a simple lemma on divergent series. We note that a similar argument is used in the proof of [27] (Theorem 9.6-1). To be self-contained, we provide the proof in Appendix A.

Lemma 3. Let $\left(a_{n}\right)_{n \in \mathbb{N}_{0}}$ be a zero convergent sequence of non-negative numbers such that

$$
\sum_{n \in \mathbb{N}_{0}} a_{n}=+\infty .
$$

Then there exists an increasing sequence of integers $m_{l}, l \in \mathbb{N}$, such that

$$
1<\sum_{n=m_{l-1}}^{m_{l}-1} a_{n}<3 .
$$

Proof. The Beurling case can be proved by making appropriate changes if the proof of [27] (Theorem 9.6-1), cf. [28]. However, since the proof for the Roumieu case contains nontrivial modifications of Zemanian's proof, we provide it here. We also consider $d=1$ for simplicity. 
(b) Let (29) hold for some $h \geq 0$, and let $f=\sum_{l \in \mathbb{N}_{0}, n \in \mathbb{Z}} c_{l, n} \psi_{l, n}$.

Let $\phi \in \mathcal{S}_{S}(\mathbb{R})$. Then we have

$$
\begin{aligned}
|\langle f, \phi\rangle|=\mid\left\langle\sum_{l \in \mathbb{N}_{0}, n \in \mathbb{Z}}\right. & \left.c_{l, n} \psi_{l, n}, \phi\right\rangle \mid \\
& \leq\left|\left\langle\sum_{l \in \mathbb{N}_{0}, n \in \mathbb{Z}} c_{l, n} e^{-h\left(\left|\frac{n}{2}\right|+|l|\right)^{1 / s}} e^{h\left(\left|\frac{n}{2}\right|+|l|\right)^{1 / s}} \psi_{l, n}, \phi\right\rangle\right| \\
& \leq \sum_{l \in \mathbb{N}_{0}, n \in \mathbb{Z}}\left|c_{l, n}\right| e^{-h\left(\left|\frac{n}{2}\right|+|l|\right)^{1 / s}} e^{h\left(\left|\frac{n}{2}\right|+|l|\right)^{1 / s}}\left|\left\langle\psi_{l, n}, \phi\right\rangle\right| \\
\leq & \left(\sum_{l \in \mathbb{N}_{0}, n \in \mathbb{Z}}\left|c_{l, n}\right|^{2} e^{-2 h\left(\left|\frac{n}{2}\right|+|l|\right)^{1 / s}}\right)^{\frac{1}{2}}\left(\sum_{l \in \mathbb{N}_{0}, n \in \mathbb{Z}}\left|\left\langle\psi_{l, n}, \phi\right\rangle\right|^{2} e^{2 h\left(\left|\frac{n}{2}\right|+|l|\right)^{1 / s}}\right)^{\frac{1}{2}} .
\end{aligned}
$$

Since $\phi \in \mathcal{S}_{\mathcal{S}}(\mathbb{R})$, by Theorem 3 (a) it follows that we can choose $h \geq 0$ such that

$$
\sum_{l \in \mathbb{N}_{0}, n \in \mathbb{Z}}\left|\left\langle\psi_{l, n}, \phi\right\rangle\right|^{2} e^{2 h\left(\left|\frac{n}{2}\right|+|l|\right)^{1 / s}}<\infty
$$

and for such choice of $h \geq 0$, by (29) it follows that

$$
\sum_{l \in \mathbb{N}_{0}, n \in \mathbb{Z}}\left|c_{l, n}\right|^{2} e^{-2 h\left(\left|\frac{n}{2}\right|+|l|\right)^{1 / s}}<\infty,
$$

and we conclude that $|\langle f, \phi\rangle|<\infty$, so that $f \in \mathcal{S}_{s}^{\prime}(\mathbb{R})$, and b) is proved.

Before we proceed to prove a) we note that (29) implies that $\left|c_{l, n}\right| \leq C e^{h\left(\left|\frac{n}{2}\right|+|l|\right)^{1 / s}}$ for every $h \geq 0$, and all $l \in \mathbb{N}_{0}, n \in \mathbb{Z}$, that is, the (double indexed) sequence $\left(\left|c_{l, n}\right| e^{-h\left(\left|\frac{n}{2}\right|+|l|\right)^{1 / s}}\right)_{l \in \mathbb{N}_{0}, n \in \mathbb{Z}}$ is bounded for every $h \geq 0$.

(a) Let $f \in \mathcal{S}_{S}^{\prime}(\mathbb{R})$ and consider $\sum_{l \in \mathbb{N}_{0}, n \in \mathbb{Z}} c_{l, n} \psi_{l, n}$ with $c_{l, n}=\left\langle f, \psi_{l, n}\right\rangle, l \in \mathbb{N}_{0}, n \in \mathbb{Z}$. If $\phi \in \mathcal{S}_{\mathcal{S}}(\mathbb{R})$ then by $(27)$ we have

$$
\begin{gathered}
\left|\left\langle\sum_{l \in \mathbb{N}_{0}, n \in \mathbb{Z}} c_{l, n} \psi_{l, n}, \phi\right\rangle\right|=\left|\sum_{l \in \mathbb{N}_{0}, n \in \mathbb{Z}} c_{l, n}\left\langle\psi_{l, n}, \phi\right\rangle\right| \\
=\left|\sum_{l \in \mathbb{N}_{0}, n \in \mathbb{Z}}\left\langle f,\left\langle\phi, \psi_{l, n},\right\rangle \psi_{l, n}\right\rangle\right|=\left|\left\langle f, \sum_{l \in \mathbb{N}_{0}, n \in \mathbb{Z}}\left\langle\phi, \psi_{l, n},\right\rangle \psi_{l, n}\right\rangle\right| \\
=|\langle f, \phi\rangle|<\infty,
\end{gathered}
$$

so that the expansion is unique and

$$
\left\langle\sum_{l \in \mathbb{N}_{0}, n \in \mathbb{Z}}\left\langle f, \psi_{l, n}\right\rangle \psi_{l, n}, \sum_{k \in \mathbb{N}_{0}, m \in \mathbb{Z}}\left\langle\phi, \psi_{k, m}\right\rangle \psi_{k, m}\right\rangle=\sum_{l \in \mathbb{N}_{0}, n \in \mathbb{Z}} \overline{a_{l, n}} c_{l, n}<\infty,
$$

where $a_{l, n}=\left\langle\phi, \psi_{l, n}\right\rangle, l \in \mathbb{N}_{0}, n \in \mathbb{Z}$.

Next we prove that the sequence $\left(e^{-k\left(\left|\frac{n}{2}\right|+|l|\right)^{1 / s}} c_{l, n}\right)_{l \in \mathbb{N}_{0}, n \in \mathbb{Z}}$ is bounded for every $k>0$.

We give the proof by contradiction: suppose that there exists $k_{0}>0$ such that the sequence $\left(e^{-k_{0}\left(\left|\frac{n}{2}\right|+|l|\right)^{1 / s}} c_{l, n}\right)_{l \in \mathbb{N} 0, n \in \mathbb{Z}}$ is unbounded. Then there exists a sequence of increasing (by components) indices $\left(l_{m}, n_{m}\right)_{m \in \mathbb{N}}$ such that

$$
e^{-k_{0}\left(\left|l_{m}\right|+\left|\frac{n_{m}}{2}\right|\right)^{1 / s}}\left|c_{l_{m}, n_{m}}\right| \geq m \quad m \in \mathbb{N} .
$$

Next we consider the sequence $\left(a_{l, n}\right)$ with the following properties
(a) $\overline{a_{l, n}} c_{l, n}=\left|a_{l, n} c_{l, n}\right|$,
(b) $\left|a_{l_{m}, n_{m}}\right|=e^{-k_{0}\left(\left|l_{m}\right|+\left|\frac{n_{m}}{2}\right|\right)^{1 / s}} \cdot \frac{1}{m}$,
(c) $a_{l, n}=0$ when $(l, n) \neq\left(l_{m}, n_{m}\right)$. 
This gives

$$
\begin{gathered}
\sum_{l \in \mathbb{N}_{0}, n \in \mathbb{Z}}\left|e^{k_{0}\left(\left|\frac{n}{2}\right|+|l|\right)^{1 / s}} a_{l, n}\right|^{2} \\
=\sum_{m \in \mathbb{N}} e^{2 k_{0}\left(\left|\frac{n_{m}}{2}\right|+\left|l_{m}\right|\right)^{1 / s}} e^{-2 k_{0}\left(\left|l_{m}\right|+\left|\frac{n_{m}}{2}\right|\right)^{1 / s}} \cdot \frac{1}{m^{2}}=\sum_{m \in \mathbb{N}} \frac{1}{m^{2}}<\infty .
\end{gathered}
$$

By Theorem 3 (b) it follows that $\phi=\sum_{l \in \mathbb{N}_{0}, n \in \mathbb{Z}} a_{l, n} \psi_{l, n} \in \mathcal{S}_{S}(\mathbb{R})$, so that $\sum_{l \in \mathbb{N}_{0}, n \in \mathbb{Z}} \bar{a}_{l, n} c_{l, n}<\infty$. On the other hand,

$$
\sum_{l \in \mathbb{N}_{0}, n \in \mathbb{Z}} \overline{a_{l, n}} c_{l, n}=\sum_{l \in \mathbb{N}_{0}, n \in \mathbb{Z}}\left|a_{l, n} c_{l, n}\right|=\sum_{m \in \mathbb{N}_{0}} e^{-k_{0}\left(\left|l_{m}\right|+\left|\frac{n_{m}}{2}\right|\right)^{1 / s}} \cdot \frac{1}{m}\left|c_{l, n}\right| \geq \sum_{m \in \mathbb{N}_{0}} 1=\infty,
$$

which gives the contradiction. $k>0$.

Thus, we conclude that the sequence $\left(e^{-k\left(\left|\frac{n}{2}\right|+|l|\right)^{1 / s}} c_{l, n}\right)_{l \in \mathbb{N}_{0}, n \in \mathbb{Z}}$ is bounded for every

Finally, we prove that (29) holds for every $h>0$. Again we give the proof by contradiction. Suppose that there exists $h_{0}>0$ such that

$$
\sum_{l \in \mathbb{N}_{0}, n \in \mathbb{Z}} b_{l, n}=\sum_{l \in \mathbb{N}_{0}, n \in \mathbb{Z}}\left|c_{l, n}\right|^{2} e^{-2 h_{0}\left(\left|\frac{n}{2}\right|+|l|\right)^{1 / s}}=\infty
$$

Since $\left(e^{-h\left(\left|\frac{n}{2}\right|+|l|\right)^{1 / s}} c_{l, n}\right)_{l \in \mathbb{N}_{0}, n \in \mathbb{Z}}$ is bounded for every $h>0$, then it follows that $\left(b_{l, n}\right)$ is a zero convergent sequence (e.g., by taking $h=h_{0}$ ). By Lemma 3 it follows that there is an increasing sequence of indices $\left(l_{m}, n_{m}\right)_{m \in \mathbb{N}}$ such that

$$
1<\sum_{j=l_{m-1}}^{l_{m}-1} \sum_{k=n_{m-1}}^{n_{m}-1}\left|c_{j, k}\right|^{2} e^{-2 h_{0}\left(\left|\frac{k}{2}\right|+|j|\right)^{1 / s}}<3 .
$$

By choosing

$$
a_{j, k}=c_{j, k} e^{-2 h_{0}\left(\left|\frac{k}{2}\right|+|j|\right)^{1 / s}} \frac{1}{m}, \quad j=l_{m-1}, \ldots, l_{m}-1, \quad k=n_{m-1}, \ldots, n_{m}-1,
$$

we obtain

$$
\sum_{j=l_{m-1}}^{l_{m}-1} \sum_{k=n_{m-1}}^{n_{m}-1}\left|a_{j, k}\right|^{2} e^{2 h_{0}\left(\left|\frac{k}{2}\right|+|j|\right)^{1 / s}}=\sum_{j=l_{m-1}}^{l_{m}-1} \sum_{k=n_{m-1}}^{n_{m}-1}\left|c_{j, k}\right|^{2} e^{-2 h_{0}\left(\left|\frac{k}{2}\right|+|j|\right)^{1 / s}} \frac{1}{m^{2}}<\frac{3}{m^{2}},
$$

for every $m \in \mathbb{N}$, where we used (31). Thus,

$$
\begin{gathered}
\sum_{l \in \mathbb{N}_{0}, n \in \mathbb{Z}}\left|a_{l, n}\right|^{2} e^{2 h_{0}\left(\left|\frac{n}{2}\right|+|l|\right)^{1 / s}} \\
=\sum_{j<l_{0}} \sum_{k<n_{0}}\left|a_{l, n}\right|^{2} e^{2 h_{0}\left(\left|\frac{n}{2}\right|+|l|\right)^{1 / s}}+\sum_{j \geq l_{0}} \sum_{k \geq n_{0}}\left|a_{l, n}\right|^{2} e^{2 h_{0}\left(\left|\frac{n}{2}\right|+|l|\right)^{1 / s}} \\
<C+\sum_{m \in \mathbb{N}} \frac{3}{m^{2}}<\infty .
\end{gathered}
$$

By Theorem 3 (b) it follows that $\sum_{l \in \mathbb{N}_{0}, n \in \mathbb{Z}} a_{l, n} \psi_{l, n} \in \mathcal{S}_{s}(\mathbb{R})$, and therefore

$$
\sum_{l \in \mathbb{N}_{0}, n \in \mathbb{Z}} \overline{a_{l, n}} c_{l, n}<\infty .
$$


However, by using the left hand side inequality in (31) we obtain

$$
\sum_{j=l_{m-1}}^{l_{m}-1} \sum_{k=n_{m-1}}^{n_{m}-1}\left|a_{j, k} c_{j, k}\right|=\sum_{j=l_{m-1}}^{l_{m}-1} \sum_{k=n_{m-1}}^{n_{m}-1}\left|c_{j, k}\right|^{2} e^{-2 h_{0}\left(\left|\frac{k}{2}\right|+|j|\right)^{1 / s}} \frac{1}{m} \geq \frac{1}{m}
$$

for each $m \in \mathbb{N}$, so that

$$
\sum_{l \in \mathbb{N}_{0}, n \in \mathbb{Z}} \overline{a_{l, n}} c_{l, n}=\sum_{l \in \mathbb{N}_{0}, n \in \mathbb{Z}}\left|a_{l, n} c_{l, n}\right| \geq \sum_{l \geq l_{0}, n \geq n_{0}}\left|a_{l, n} c_{l, n}\right| \geq \sum_{m \in \mathbb{N}} \frac{1}{m}=\infty .
$$

This is a contradiction with (32). We conclude that the assumption (30) cannot hold. Therefore

$$
\sum_{l \in \mathbb{N}_{0}, n \in \mathbb{Z}}\left|c_{l, n}\right|^{2} e^{-2 h\left(\left|\frac{n}{2}\right|+|l|\right)^{1 / s}}<\infty
$$

for every $h>0$, which completes the proof.

\section{Alternative Proof via Modulation Spaces}

Modulation spaces, originally introduced by Feichtinger in [29], are recognized as an appropriate family of spaces when dealing with problems of time-frequency analysis, see $[17,18,29,30]$, to mention just a few references. A broader family of modulation spaces, including quasi-Banach spaces when the Lebesgue parameters $p, q$ belong to $(0,1)$ is studied in, e.g., [31].

Let there be given $\phi \in \Sigma_{1}\left(\mathbb{R}^{d}\right) \backslash 0, p, q \in[1, \infty]$ and $\omega \in \mathcal{P}_{E}\left(\mathbb{R}^{2 d}\right)$. Then the modulation space $M_{\omega}^{p, q}\left(\mathbb{R}^{d}\right)$ consists of all Gelfand-Shilov distributions $f \in \Sigma_{1}^{\prime}\left(\mathbb{R}^{d}\right)$ such that

$$
\|f\|_{M_{\omega}^{p, q}} \equiv\left(\int\left(\int\left|V_{\phi} f(x, \xi) \omega(x, \xi)\right|^{p} d x\right)^{q / p} d \xi\right)^{1 / q}<\infty
$$

(with the obvious changes if $p=\infty$ and /or $q=\infty$ ). If $p=q$ we simply write $M_{\omega}^{p}$ instead of $M_{\omega}^{p, p}$, and if $\omega=1$, then we set $M^{p, q}=M_{\omega}^{p, q}$ and $M^{p}=M_{\omega}^{p}$.

If $\omega$ is $v$-moderate, then the spaces $M_{\omega}^{p, q}$ are (quasi-)Banach spaces and different choices of $\phi \in M_{v}^{1} \backslash 0$ give rise to equivalent (quasi-)norms in (33), and so $M_{\omega}^{p, q}$ is independent on the choice of $\phi \in M_{v}^{1}$ [31] (Proposition 2.1).

For $p, q \in[1, \infty)$ and $\omega \in \mathcal{P}_{E}\left(\mathbb{R}^{2 d}\right)$ the dual of $M_{\omega}^{p, q}\left(\mathbb{R}^{d}\right)$ is $M_{1 / \omega}^{p^{\prime}, q^{\prime}}\left(\mathbb{R}^{d}\right)$, where $\frac{1}{p}+\frac{1}{p^{\prime}}$ $=\frac{1}{q}+\frac{1}{q^{\prime}}=1$.

For a given weight $\omega \in \mathcal{P}_{E}\left(\mathbb{R}^{2 d}\right)$, we put $\tilde{\omega}$ for the (double) sequence $\tilde{\omega}(n, l)=\omega\left(\frac{n}{2}, l\right)$, $(n, l) \in \mathbb{Z}^{d} \times \mathbb{N}_{0}^{d}$. By $l_{\tilde{\omega}}^{p, q}, p, q \in[1, \infty]$, we denote the space of sequences $\left(a_{l, n}\right)_{(l, n) \in \mathbb{N}_{0}^{d} \times \mathbb{Z}^{d}}$ for which the norm

$$
\left\|a_{l, n}\right\|_{l_{\tilde{\omega}^{p, q}}}=\left(\sum_{l \in \mathbb{N}_{0}^{d}}\left(\sum_{n \in \mathbb{Z}^{d}}\left|a_{l, n}\right|^{p} \tilde{\omega}(n, l)^{p}\right)^{q / p}\right)^{1 / p}
$$

is finite.

The next theorem is analogous to Theorem 1. It follows from [17] (Chapter 12.3), so we omit the proof.

Theorem 5. Let $p, q \in[1, \infty], \omega \in \mathcal{P}_{E}\left(\mathbb{R}^{2 d}\right)$, and let there be given a Wilson basis of exponential

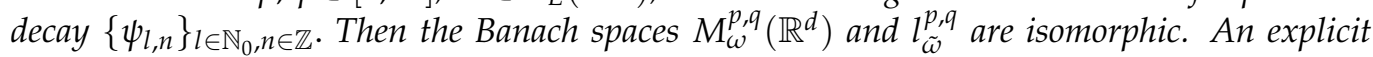
isomorphism is provided by the coefficient operator $C_{\psi}: M_{\omega}^{p, q}\left(\mathbb{R}^{d}\right) \rightarrow l_{\tilde{\omega}}^{p, q}$ given by

$$
C_{\psi} f=\left(\left\langle f, \psi_{l, n}\right\rangle\right)_{(l, n) \in \mathbb{N}_{0}^{d} \times \mathbb{Z}^{d}} .
$$


By Theorem 5 it follows that

$$
f=\sum_{l \in \mathbb{N}_{0}^{d}, n \in \mathbb{Z}^{d}}\left\langle f, \psi_{l, n}\right\rangle \psi_{l, n}
$$

with the unconditional convergence in $M_{\omega}^{p, q}\left(\mathbb{R}^{d}\right)$ if $1 \leq p, q<\infty$, and weak* convergence in $M_{1 / v}^{\infty}\left(\mathbb{R}^{d}\right)$ otherwise.

Gelfand-Shilov spaces and their dual spaces can be described as projective or inductive limits of modulation spaces as follows.

Theorem 6. Let $1 \leq p, q \leq \infty, s>1 / 2$, and set

$$
\omega_{h}(x, \omega) \equiv e^{h\left(|x|^{1 / s}+|\xi|^{1 / s}\right)}, \quad h>0, x, \xi \in \mathbb{R}^{d} .
$$

Then

$$
\begin{array}{ll}
\Sigma_{s}\left(\mathbb{R}^{d}\right)=\bigcap_{h>0} M_{\omega_{h}}^{p, q}\left(\mathbb{R}^{d}\right), & \left(\Sigma_{s}\right)^{\prime}\left(\mathbb{R}^{d}\right)=\bigcup_{h>0} M_{1 / \omega_{h}}^{p, q}\left(\mathbb{R}^{d}\right), \\
\mathcal{S}_{s}\left(\mathbb{R}^{d}\right)=\bigcup_{h>0} M_{\omega_{h}}^{p, q}\left(\mathbb{R}^{d}\right), & \left(\mathcal{S}_{s}\right)^{\prime}\left(\mathbb{R}^{d}\right)=\bigcap_{h>0} M_{1 / \omega_{h}}^{p, q}\left(\mathbb{R}^{d}\right) .
\end{array}
$$

Proof. The proof is well known, see e.g., [21] (Theorem 3.9) and [32].

However, we may give a simple independent proof based on Theorem 2 when $p=$ $q=2$. Namely, if we put $\omega_{1}(x)=e^{h|x|^{1 / s}}$ and $\omega_{2}(\xi)=e^{h|\xi|^{1 / s}}, x, \xi \in \mathbb{R}^{d}, s>1$, and $h \geq 0$, then by definition we have

$$
M_{1 \otimes \omega_{2}}^{2}\left(\mathbb{R}^{d}\right)=\mathcal{C} o Y^{h, s}\left(\mathbb{R}^{d}\right) \quad \text { and } \quad M_{\omega_{1} \otimes 1}^{2}\left(\mathbb{R}^{d}\right)=\mathcal{F} \mathcal{C} o Y^{h, s}\left(\mathbb{R}^{d}\right),
$$

see also [14], and the claim follows directly from Theorem 2. The same conclusion for general $1 \leq p, q \leq \infty$ holds from embedding properties of modulation spaces and certain equivalence properties of norms for Lebesgue spaces. We omit details, and refer the reader to, e.g., [32].

As noted by Gröchenig, the isomorphism in Theorem 5 can be formulated in a different mathematical language. For example, in combination with Theorem 6 we conclude that there exists a tame isomorphism between FS spaces $\Sigma_{S}\left(\mathbb{R}^{d}\right)$ and $\cap_{h>0} l_{\tilde{\omega}_{h}}^{p, q}$ and between LS spaces $\mathcal{S}_{S}\left(\mathbb{R}^{d}\right)$ and $\cup_{h>0} l_{\tilde{\omega}_{h}}^{p, q}$. We refer to [12] for the precise definition of tame isomorphisms and related considerations in the context of Hermite functions expansions instead of Wilson bases.

Now we can present an alternative proof of our main results.

Proof. (Alternative proof of Theorems 3 and 4) We give the proof for the Roumieu case $\mathcal{S}_{S}\left(\mathbb{R}^{d}\right)$ and $\left(\mathcal{S}_{S}\right)^{\prime}\left(\mathbb{R}^{d}\right)$. The Beurling case can be proved by using similar arguments.

Let $f \in \mathcal{S}_{s}\left(\mathbb{R}^{d}\right)$. By Theorem 6 it follows that there exists $h>0$ such that $f \in M_{\omega_{h}}^{2}\left(\mathbb{R}^{d}\right)$, where $\omega_{h}$ is given by (35). Now, Theorem 5 implies that

$$
f=\sum_{l \in \mathbb{N}_{0}^{d}, n \in \mathbb{Z}^{d}} c_{l, n} \psi_{l, n}, \quad \text { where } \quad c_{l, n}=\left\langle f, \overline{\psi_{l, n}}\right\rangle,
$$

and $c_{l, n} \in l_{\tilde{\omega}_{h}}^{2,2}$,i.e.,

$$
\sum_{l \in \mathbb{N}_{0}^{d}, n \in \mathbb{Z}^{d}}\left|c_{l, n}\right|^{2} e^{2 h(|n / 2|+|l|)^{1 / s}}<\infty, \quad \text { for some } h \geq 0,
$$

which proves Theorem 3(a). 
The converse part follows from the fact that under the assumption of Theorem 3(b) we have

$$
\sum_{l \in \mathbb{N}_{0}^{d}, n \in \mathbb{Z}^{d}} c_{l, n} \psi_{l, n} \in M_{\omega_{h}}^{p, q}
$$

for some $h>0$. Therefore, by Theorem 6 it follows that the sum represents a unique element $f \in \mathcal{S}_{s}\left(\mathbb{R}^{d}\right)$, and since the Wilson basis is an ONB it follows that $c_{l, n}=\left\langle f, \overline{\psi_{l, n}}\right\rangle$, and we are done.

Theorem 4 follows by duality.

\section{Discussion}

The proof of Theorems 3 and 4 given in Section 4 does not use any reference to the coorbit space theory employed in Section 3. It essentially used representation of modulation spaces by means of Wilson bases, which follows from the relation between Wilson bases and Gabor frames, cf. [17]. Another ingredient is Theorem 6, which can be proved without the coorbit space theory, see [21] (Theorem 3.9).

On the other hand, the proof presented in Section 3 is based on direct estimates, and does not rely on other results, apart from checking whether the construction of Wilson bases fits well to the general theory of coorbit spaces, which is done in $[2,14]$.

We note that in the background of both proofs are decay properties of the STFT and the exponential decay property of the considered Wilson bases. Therefore, the techniques from the present paper can be modified to include other time-frequency representations and also more general (for example anisotropic) Gelfand-Shilov type spaces, cf. [23].

Funding: This research received no external funding.

Institutional Review Board Statement: Not applicable.

Informed Consent Statement: Not applicable.

Data Availability Statement: Not applicable.

Acknowledgments: The work is partially supported by projects "Localization in Phase space: theoretical, numerical and practical aspects" No. 19.032/961-103/19 MNRVOID Republic of Srpska, TIFREFUS Project DS 15, and MPNTR of Serbia Grant No. 451-03-9/2021-14/200125. We would like to thank the referees for their recommendations, which helped to improve the quality of the paper.

Conflicts of Interest: The authors declare no conflict of interest. The funders had no role in the writing of the manuscript or in the decision to publish the results.

\section{Appendix A}

Since $\sum_{n=1}^{\infty} a_{n}=\infty$, by a small abuse of notation, we consider the subsequence so that $a_{n}>0, \forall n \in \mathbb{N}$. Consider the partial sum $s_{m_{0}}=\sum_{n=1}^{m_{0}} a_{n}=M$, where $m_{0}$ is chosen such that $a_{n}<1$ for all $n \geq m_{0}-1$. Then we have:

$$
\begin{gathered}
M-1 \leq s_{m_{0}-1}<M \leq s_{m_{0}}<M+1, \\
s_{m_{0}}-s_{m_{0}-1}=a_{m_{0}}<1 \quad \text { and } \quad s_{m_{0}-1}-s_{m_{0}-2}=a_{m_{0}-1}<1 .
\end{gathered}
$$

Moreover, $s_{m_{0}+1}<M+2$.

Now choose $m_{1} \in \mathbb{N}$ as the minimal index such that

$$
s_{m_{1}} \geq M+3
$$

Thus, we have $M+3>s_{m_{1}-1} \geq M+2$, and $m_{1}-1>m_{0}$ Moreover, $s_{m_{1}}=s_{m_{1}-1}+$ $a_{m_{1}}<M+4$.

Therefore, we have the following situation:

$$
M \leq s_{m_{0}}<M+1<M+2 \leq s_{m_{1}-1}<M+3 \leq s_{m_{1}}<M+4,
$$


wherefrom

$$
1<s_{m_{1}-1}-s_{m_{0}}<3 .
$$

We continue as follows: choose $m_{2} \in \mathbb{N}$ such that $m_{2}-1>m_{1}$ and

$$
M+3 \leq s_{m_{1}}<M+4<M+5 \leq s_{m_{2}-1}<M+6 \leq s_{m_{2}}<M+7 .
$$

This gives $1<s_{m_{2}-1}-s_{m_{1}}<3$.

By choosing $m_{l}, l \geq 3$, in an analogous way, we obtain an increasing sequence of integers such that

$$
1<\sum_{n=m_{l-1}}^{m_{l}-1} a_{n}<3,
$$

which proves the claim.

The same arguments show that for any given $\varepsilon>0$ there exists an increasing sequence of integers $m_{l}, l \in \mathbb{N}$, such that

$$
\frac{\varepsilon}{3}<\sum_{n=m_{l-1}}^{m_{l}-1} a_{n}<\varepsilon .
$$

\section{References}

1. Daubechies, I.; Jaffard, S.; Journé, J. A Simple Wilson Orthonormal Basis With Exponential Decay. SIAM J. Math. Anal. 1991, 22, 554-572. [CrossRef]

2. Feichtinger, H.G.; Gröchenig, K.; Walnut, D. Wilson Bases and Modulation Spaces. Math. Nachr. 1992, 155, 7-17. [CrossRef]

3. Pilipović, S.; Teofanov, N. Pseudodifferential operators on ultra-modulation spaces. J. Funct. Anal. 2004, 208, 194-228. [CrossRef]

4. Tachizawa, K. The boundedness of pseudodifferential operators on modulation spaces. Math. Nachr. 1994, 168, $263-277$. [CrossRef]

5. Chassande-Mottin, E.; Jaffard, S.; Meyer, Y. Des ondelettes pour détecter les ondes gravitationnelle. Gaz. Math. 2016, 148, 61-64

6. Necula, V.; Klimenko, S.; Mitselmakher, G. Transient analysis with fast Wilson-Daubechies time-frequency transform. J. Phys. Conf. Ser. 2012, 363, 012032. [CrossRef]

7. Bownik, M.; Jakobsen, M.S.; Lemvig, J.; Okoudjou, K.A. On Wilson bases in $L^{2}\left(\mathbb{R}^{d}\right)$. SIAM J. Math. Anal. 2017, 49, 3999-4023. [CrossRef]

8. Tachizawa, K. The pseudodifferential operators and Wilson bases. J. Math. Pures Appl. 1996, 75, 509-529.

9. Gelfand, I.M.; Shilov, G.E. Generalized Functions, II; Academic Press: New York, NY, USA, 1968.

10. Gramchev, T. Gelfand-Shilov Spaces: Structural Properties and Applications to Pseudodifferential Operators in $\mathbb{R}^{n}$, in Quantization, PDEs, and Geometry, 1-68. Oper. Theory Adv. Appl. 251; Birkhäuser/Springer: Cham, Switzerland, 2016.

11. Toft, J. Images of function and distribution spaces under the Bargmann transform. J. Pseudo-Differ. Oper. Appl. 2017, 8, 83-139. [CrossRef]

12. Langenbruch, M. Hermite functions and weighted spaces of generalized functions. Manuscripta Math. 2006, 119, 269-285. [CrossRef]

13. Pilipović, S. Tempered Ultradistributions. Boll. Un. Mat. Ital. 1988, 7, 235-251.

14. Pilipović, S.; Teofanov, N. Wilson bases and ultramodulation spaces. Math. Nach. 2002, 242, 179-196. [CrossRef]

15. Feichtinger, H.G.; Gröchenig, K. Banach Spaces Related to Integrable Group Representations and Their Atomic Decompositions, I. J. Funct. Anal. 1989, 86, 307-340. [CrossRef]

16. Feichtinger, H.G.; Gröchenig, K. Banach Spaces Related to Integrable Group Representations and Their Atomic Decompositions. Part II. Mon Hefte Math. 1989, 108, 129-148. [CrossRef]

17. K. Gröchenig. Foundations of Time-Frequency Analysis; Birkhäuser: Boston, MA, USA, 2001.

18. Cordero, E.; Rodino, L. Time-Frequency Analysis of Operators; Studies in Mathematics, 75; De Gruyter: Berlin, Germany, 2020.

19. Wilson, K.G. Generalized Wannier Functions; 1987; Unpublished Manuscript.

20. Gröchenig, K. Weight functions in time-frequency analysis. In Pseudodifferential Operators: Partial Differential Equations and Time-Frequency Analysis; AMS: Providence, RI, USA, 2007; pp. 343-366.

21. Toft, J. The Bargmann transform on modulation and Gelfand-Shilov spaces, with applications to Toeplitz and pseudo-differential operators. J. Pseudo-Differ. Oper. Appl. 2012, 3, 145-227. [CrossRef]

22. Dahlke, S.; Häuser, S.; Teschke, G.S.G. Shearlet Coorbit Theory. In Harmonic and Applied Analysis; Applied and Numerical Harmonic Analysis; Dahlke, S., Mari, F.D., Grohs, P., Labate, D., Eds.; Birkhäuser: Cham, Switzerland, 2015; Volume 68, pp. 83-147.

23. Teofanov, N. Gelfand-Shilov spaces and localization operators. Funct. Anal. Approx. Comput. 2015, 2, 135-158.

24. Gröchenig, K.; Zimmermann, G. Spaces of test functions via the STFT. J. Funct. Spaces Appl. 2004, 2, 25-53. [CrossRef] 
25. Kamiński, A.; Perišić, D.; Pilipović, S. On Various Integral Transformations of Tempered Ultradistributions. Demonstr. Math. 2000, XXXIII, 641-655. [CrossRef]

26. Cordero, E.; Pilipović, S.; Rodino, L.; Teofanov, N. Quasianalytic Gelfand-Shilov spaces with applications to localization operators. Rocky Mt. J. Math. 2010, 40, 1123-1147. [CrossRef]

27. Zemanian, A.H. Generalized Integral Transformations; John Wiley and Sons: New York, NY, USA, 1968.

28. Teofanov, N. Ultramodulation Spaces and Pseudodifferential Operators; Endowment Andrejević: Belgrade, Serbia, 2003.

29. Feichtinger, H.G. Modulation Spaces on Locally Compact Abelian Groups; Technical Report; University of Vienna: Vienna, Austria, 1983; Also in Wavelets and Their Applications, 99-140; Allied Publishers Private Limited: New Dehli, India, 2003.

30. Bényi, A.; Okoudjou, K. Modulation Spaces With Applications to Pseudodifferential Operators and Nonlinear Schrödinger Equations; Springer Science+Business Media, LLC: New York, USA, 2020.

31. Toft, J. Continuity of Gevrey-Hörmander pseudo-differential operators on modulation spaces. J. Pseudo-Differ. Oper. Appl. 2019, 10, 337-358. [CrossRef]

32. Teofanov, N. Modulation spaces, Gelfand-Shilov spaces and pseudodifferential operators. Sampl. Theory Signal Image Process. 2006, 5, 225-242. [CrossRef] 